\title{
BIOMETRIC SYSTEMS BASED ON ECG USING ENSEMBLE EMPIRICAL MODE DECOMPOSITION AND VARIATIONAL MODE DECOMPOSITION
}

\author{
Sugondo Hadiyoso*, Inung Wijayanto, Achmad Rizal, Suci Aulia \\ Telkom University, Bandung, Indonesia
}

Electrocardiogram (ECG) based biometric is challenging to be developed with the aim of high-security access. This biometric system is more difficult to falsify, compared to the conventional biometric systems. From previous proposed studies, there is still a gap to improve the accuracy of the system. Therefore in this study, a new protocol is proposed to improve the performance of the ECG biometric system compared to previously reported studies. This study decomposes the ECG signals using a method based on empirical mode decomposition (EMD) based, which are Variational Mode Decomposition (VMD) and Ensemble Empirical Mode Decomposition (EEMD). These two methods are the development of the EMD method to overcome one main problem of EMD. That is, the EMD method generates oscillations with the same time scales, which stored in different decomposition levels. A private ECG dataset, recorded using one lead ECG signal from 11 subjects, is used in this study. ECG signals from each person are then segmented into ten windows to become training data and test data. VMD and EEMD methods are used to decompose ECG signals into five sub-signals. Feature extraction based on statistical calculations is applied at each level of decomposition to obtain the characteristics of the ECG signal. Mean, variance, skewness, kurtosis, and entropy are evaluated as predictors. Support vector machines and 10-fold cross-validation are used to validate the performance of the proposed method. Our simulations demonstrate that the proposed method outperforms several previous studies and achieves an accuracy of up to $98.2 \%$.

Key words: ECG, identification, VMD, EEMD, statistical

\section{INTRODUCTION}

Biometrics has been widely used for identification, authentication, or security system applications. The biometric concept consists of psychological and behavioral modality. Physical biometric systems such as fingerprints and iris-based are the most commonly applied. However, both of them tend to be easily falsified so that the potential for misuse [1], for example, for criminal acts. Therefore, many studies have begun to lead to the search for new biometric approaches. One biometric modality that has recently received attention is the electrocardiogram (ECG) based on biometrics. This is based on the hypothesis that individuals have unique ECG waveforms [2].

Biometric research with ECG modalities has been carried out by previous studies in both simulation and implementation that were tested offline or in real-time. The study by Biel et al. [3] conducted a biometric simulation based on ECG using a 12-lead ECG standard. From this study, it is possible to extract features from one lead only. Similar research by Vessela [4], simulates 12 leads ECG-based biometric. Another study by Jekova [5], simulates personal identification using the ECG and even analyzes the influence of the personal health status on accuracy. However, the proposed studies use a 12 lead ECG system, so it tends to be complex.

Many previous studies propose various methods of feature extraction, including analysis in the time domain, frequency, time-frequency, and wavelet. Wavelet-based analysis methods on ECG biometric simulations have been proposed in research [6-11]. Another proposed method for feature extraction on ECG biometrics applications is the frequency-based approach. Analysis of the frequency domain based on the Fourier transform has been conducted in research [12-13]. Other researchers propose the time-frequency domain method for feature extraction, as reported in [14-15]. Another method based on template analysis has been proposed in research [16], in its simulations resulting true acceptance rate (TAR) $<90 \%$. Recently a deep neural network approach was proposed in ECG biometric systems as reported in the study [17]. However, this requires large memory resources to process the data that it might be difficult if implemented on low-cost computers.

Each of the proposed studies achieves high accuracy. This can strengthen the hypothesis that ECG has the opportunity to become a biometric modality in the future. Although studies show good performance, it cannot be compared to one another, because there are differences in the number of ECG leads, ECG datasets, and devices used. At least the results of this research have strengthened one study to another. Furthermore, the proposed methods can be used as a reference in the development of ECG-based in the near future.

In previous studies, sample entropy (SampEn) and Hjorth descriptors were proposed for the feature extraction 
method in ECG-based biometric [18]. The accuracy result was $93.8 \%$. Another study measured the entropy on wavelet decomposition signals. The accuracy result was 71.8\% [19]. The empirical mode decomposition (EMD) method with statistical measurements has also been proposed in previous studies [20]. The accuracy result was 93.6\%, not outperform compared with study [18]. From the previous proposed studies, there is still a gap and also opportunities to improve accuracy. Since the EMD method has a drawback which generates data at the same time scale but is stored at a different level of decomposition.

This study proposes an ECG biometric method using Ensemble Empirical Mode Decomposition (EEMD) and Variational Mode Decomposition (VMD) combined with statistical analysis. This study aims to improve the performance of our previous studies in [18-20]. The ECG signals are decomposed into five levels using EEMD and VMD. Statistical analysis is used to get the characteristics of each decomposed signal. The proposed method in this research is tested and evaluated on a private dataset with a higher number of subjects, consisting of 11 subjects. Each subject has 10 ECG signals. Thus 110 ECG signals are simulated to become the training and test data. The validation and performance tests are carried out using Support Vector Machine (SVM) and 10-fold cross-validation. Some SVM kernels have been used with the aim of finding the best performance. These simulations show that the proposed method outperforms several previous studies and achieves $98.2 \%$ of maximum accuracy using linear and quadratic SVM.

The remaining section of the paper is organized as follows: Section 2 explains the data collection and proposed method, including feature extraction and classification. Section 3 describes the results and analysis of the study, followed by a discussion. Meanwhile, the conclusions of this study presented in section 4.

\section{MATERIALS AND METHODS}

\section{Data collection}

ECG signals are recorded using a one-lead digital ECG device that we have developed [21]. This study used the same dataset with our previous research in [19], [20], which consists of 11 subjects. ECG signal leads refer to the Einthoven triangle tapping technique. This digital ECG has a resolution of 10 bits with a sampling frequency of $100 \mathrm{~Hz}$. A sampling frequency of $100 \mathrm{~Hz}$ is a recommendation in the study of [22] for heart rate analysis. At the time of recording, the subject is relaxed and sat on a chair. The total recording duration of each person is one minute The ECG signal data stream is then stored in .txt format to be analyzed and simulated. Figure 1 shows an example of a raw ECG signal from one of the subjects.

This section describes the proposed method for the ECG biometric system. Figure 2 shows the general process of the proposed method. In the pre-processing stage, the raw ECG signal is filtered to reject a large amount of noise. EEMD and VMD then decompose Noise-free signals into five levels. The next process is feature extraction by calculating statistical parameters for each decomposed signal. The performance of the proposed method is tested using SVM and cross-validation. The success parameter of this research is the value of the accuracy of the system in person identifying.

\section{Signal pre-processing}

Pre-processing is intended to reject low and high-frequency noise in the form of baseline wandering and muscle noise. In this process, signal normalization also carried out to avoid large deviations that may reduce system performance. Pre-processing is carried out by a high pass filter (HPF) with a cut-off frequency of $0.5 \mathrm{~Hz}$ and a low pass filter (LPF) with a cut-off frequency of $50 \mathrm{~Hz}$ [23]. The pre-processed signal, which is noisefree, is shown in Figure 3.

\section{Signal decomposition}

Signal decomposition aims to obtain essential information from the observed signal so that the characteristics of the ECG signal from each person can be calculated in more detail. The most common approaches for signal decomposition are the time domain and time-frequency domain.One of the popular time domain based decom-

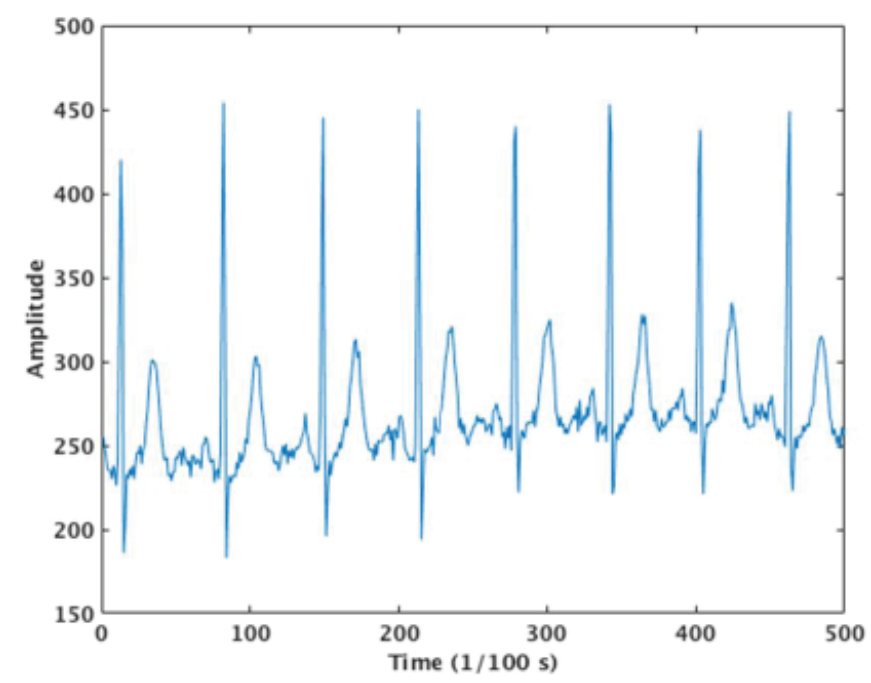

Figure 1: Raw ECG signal from one of subjects

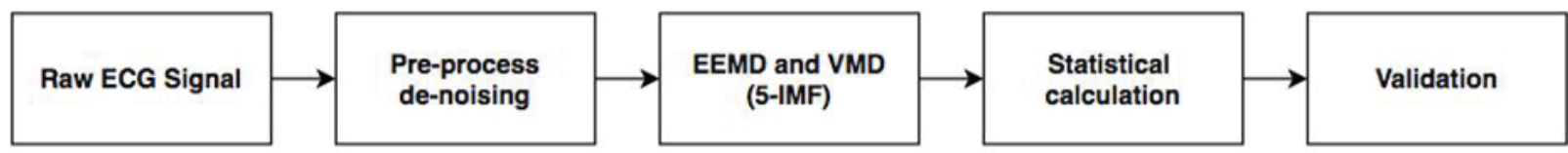

Figure 2: Proposed method for ECG biometric system 


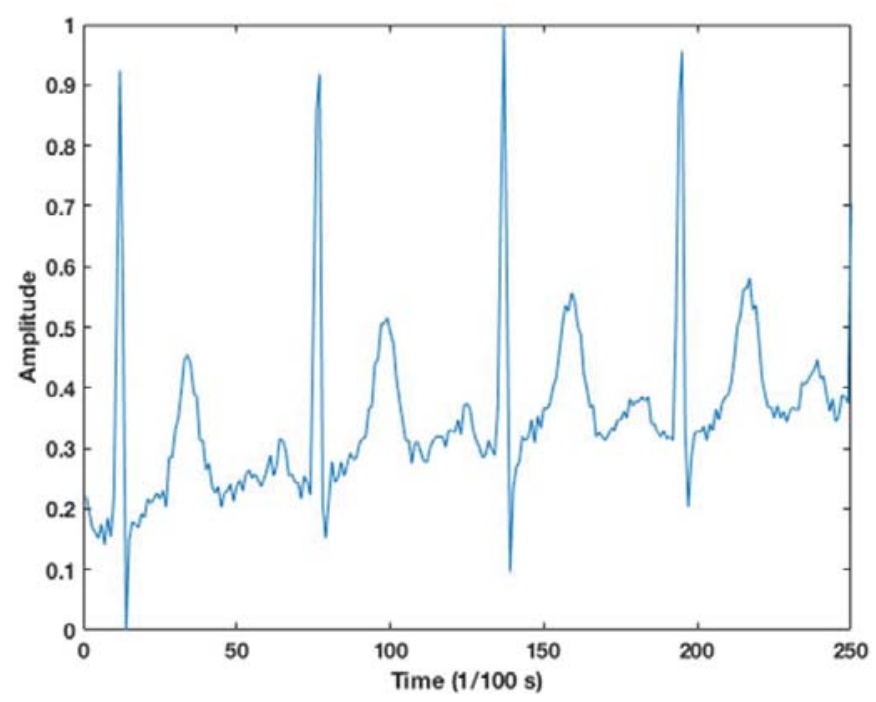

Figure 3: Pre-processed signal

position methods is EMD. Meanwhile the time-frequency domain based method is wavelet transform [24]. In this study, two signal decomposition methods, namely EEMD and $\mathrm{VMD}$, have been simulated. The signal decomposition analyzes IMF-1 to IMF-5. The resulting decomposition can be up to IMF-7 for each observed ECG waveform, but at that level, the decomposition results tend to be monotonous. Following is a description of the EEMD and VMD methods.

\section{Ensemble Empirical Mode Decomposition (EEMD)}

EEMD is the development of empirical mode decomposition (EMD), which was first introduced by Hilbert Huang in 1998 [25]. EEMD is claimed to be able to overcome the weaknesses of EMD [26]. The weakness of EMD is that oscillations with the same time scale are stored in different decomposition levels or vice versa [27]. This condition is ineffective, and the intrinsic information signals become more difficult to determine. The report then becomes our reason for optimizing our previous study [20], which used the EMD method. EEMD has the ability to scale better. This has been proven through trials with the addition of white noise to the signal [28]. Each decomposition produced by the EEMD does not show a relationship between one another. EEMD is able to compensate for noise better because one of the parameters calculated is noise amplitude (A). EEMD can be applied according to the following algorithm:

1. Add white noise to the original signal according to the following equation.

$X(t)+u_{n}(t)$, forn $=1,2, \ldots, N$

Where

$X(t) \quad=$ original signal

$u_{n}(t)=$ white noise

$Y_{n}(t)=$ signal with white noise according to the ensemble number $(\mathrm{N})$.
2. Signal decomposition of $Y_{n}(t)$ into several IMF with the residue.

$Y_{n}(t)=\sum_{m=1}^{M-1} I M F_{m}^{(n)}(t)+r_{M}^{(n)}(t)$

M-1 is the total amount of IMF decomposed from, $I M F_{m}^{(n)}$ is the $m^{\text {th }}$ level/mode and $r_{M}^{(n)}$ is the remainder obtained in the $n^{\text {th }}$ experiment.

3. Repeating the first and second steps using different white noise added to the original signal.

4. Calculate the average $I M F_{m}^{\text {ave }}$ of severalensembles $(N)$ using the following equation.

$I M F_{m}^{a v e}(t)=\frac{1}{N} \sum_{n-1}^{N} I M F_{m}^{(n)}(t)$

\section{Variational Mode Decomposition (VMD)}

Variational mode decomposition (VMD) is a decomposition method of non-stationary signals. It decomposes the signal into several components that are entirely intrinsic and non-recursive [29]. VMD is developed to overcome EMD deficiencies, including backward error correction, noise sensitivity, and the selection of predefined filter bank boundaries. The VMD method adaptively calculates related bands and overcomes the presence of noise so that it can decompose the input signal more efficiently. The VMD method has become a popular tool, one of which is in biomedical signal processing. In a simple definition, VMD decomposes the original signal into a band-limited ensemble mode [30]. To assess the bandwidth of one-dimensional signal $s$ with a mode $u_{k}$ can be done by following these steps:

- Compute the analytic signal associating with $u_{k}$ to obtain a unilateral frequency spectrum by using Hilbert transform,

- The frequency spectrum of the mode is shifted to baseband. This process is done by mixing the exponential to the respective estimated center frequency,

- Calculate the bandwidth using H1 Gaussian smoothness of the demodulated signal.

The constrained variational problem of VMD is defined as [29]:

$\min _{\left\{u_{k}\right\},\left\{\omega_{k}\right\}}\left\{\sum_{k=1}^{K}\left\|\partial_{t}\left[\left(\delta(t)+\frac{j}{\pi t}\right) * u_{k}(t)\right] e^{-j \omega_{k} t}\right\|_{2}^{2}\right\}$

s.t. $\sum_{k=1}^{K} u_{k}=f$

Here, $f$ is the input signal, $u_{k}$ is the subcomponent's discrete numbers or the modes which, during the reproducing the input, have specific sparsity properties. The sub-signals are compact around the center pulsation and 
said as a band-limited intrinsic mode function (BLIMF). The expected number of BLIMF is defined as $K$ while the center frequencies of BLIMF and short-hand notations are expressed with $u_{k}$ and $\omega_{k}$.

The results of decomposition both from EEMD and VMD are then analyzed statically as a signal feature set. The statistical parameters are mean, variance, skewness, kurtosis, and entropy. The definition of these five parameters is explained in the previous study [20].

\section{Support Vector Machine (SVM)}

Support Vector Machine (SVM) is a discriminative classifier developed by Cortes and Vapnik in 1995 [31]. Basically, SVM is used for solving a two-classes classification task [32]. The main concept of SVM is by mapping the input vector into a very high dimension feature space. The next process is by creating an imaginary plane which called as hyperplane. This hyperplane then used to separate the data into two groups. SVM is an effective tool for classification problems and pattern recognition. It is very applicable in many fields, including image processing [33], [34], bio-signal analysis such as electrocardiogram [35], [36] and electroencephalogram [37-39]. Two types of SVM are used in this study.

\section{Linear Support Vector Machine (SVM)}

To optimize the distance between the nearest data and the hyperplane, an optimization algorithm is needed. For a group of data $X$ such as expressed in Equation 5 .

$X=\left\{\left(\vec{a}_{i}, b_{i}\right) \mid \vec{a}_{i} \in i^{p}, b_{i} \in\{-1,1\}\right\}_{i=1}^{n}$

Here, the value of $b_{i}$ is -1 or 1 . The number of training data is denoted with $n$. Therefore, for every $\vec{a}_{i}$, the hyperplane can be expressed in Equation 6.

$\vec{w} \cdot \vec{a}-x=0$

Here, the vector to the hyperplane is denoted as $\vec{w}$. If the data can be separated linearly, the hyperplane is expressed in Equation 7 and Equation 8.

$$
\begin{aligned}
& \vec{w} \cdot \vec{a}-x=1 \\
& \vec{w} \cdot \vec{a}-x=-1
\end{aligned}
$$

On the other hand, if the data cannot be separated linearly, then the calculation of the hyperplane is needed to be adjusted by using Equation 9 .

$$
\min 1 / 2\|\vec{w}\|^{2}+T \sum_{i=1}^{k} \varepsilon_{i}
$$

Here, the $T$ is the trade-off parameter between the error of the training set and the separation of the classes. The $\varepsilon$ is the set of slack variables. The main purpose is to find the minimum distance between two hyperplanes $2\|\bar{w}\|)$ by minimizing the $\|\vec{w}\|$.

\section{Nonlinear Support Vector Machine (SVM)}

Another approach for the SVM classifier is by using a kernel trick to extend to a non-linear surface, such as proposed by Boser et al. [40]. The original space data can be shifted to the higher dimensional space by using non-linear functions. They are polynomial and Gaussian function (radial basis function). This study used the polynomial functions, which consist of the quadratic and cubic functions. The function is expressed in Equation 10. Here, for quadratic function the $d=2$, and for cubic function, the $d=3$.

$$
k\left(a_{i}, b_{i}\right)=\left(a_{i}, b_{i}+1\right)^{d}
$$

\section{RESULTS AND DISCUSSION}

Figure 4 and Figure 5 shows an example of the decomposition results (IMF-1 to IMF-5) in two different subjects. Visually there is a difference in each of the decomposition signals between the two subjects, which are observed. The EEMD and VMD results show that there are differences in signal form between the two subjects. Statistical features are then calculated in all decomposition signals producing 25 feature vectors. Figure 6 shows the average value of each statistical parameter of each subject. Significance differences test in each feature for each IMF was also observed using one-way ANOVA. Differences between individual ECGs are considered to have statistical significance if the $p$-value $<0.05$. The results of the significance tests are presented in Table 1. Each IMF has

Table 1: Validation results of the proposed method

\begin{tabular}{|c|c|c|c|c|c|}
\hline \multirow{2}{*}{ IMF } & Mean & Variance & Skewness & Kurtosis & Entropy \\
\cline { 2 - 6 } & \multicolumn{5}{|c|}{-value } \\
\hline IMF-1 & $0.000^{\star}$ & $0.030^{\star}$ & 0.108 & $0.003^{\star}$ & $0.003^{\star}$ \\
\hline IMF-2 & $0.005^{\star}$ & 0.059 & $0.002^{\star}$ & $0.000^{\star}$ & $0.007^{*}$ \\
\hline IMF-3 & $0.043^{\star}$ & 0.075 & 0.146 & 0.173 & 0.175 \\
\hline IMF-4 & $0.008^{*}$ & $0.007^{\star}$ & 0.065 & $0.002^{\star}$ & $0.000^{*}$ \\
\hline IMF-5 & 0.088 & 0.056 & 0.053 & $0.002^{\star}$ & 0.051 \\
\hline * indicates $\mathrm{p}<0.05$ \\
\hline
\end{tabular}


IMF-1 to IMF-5
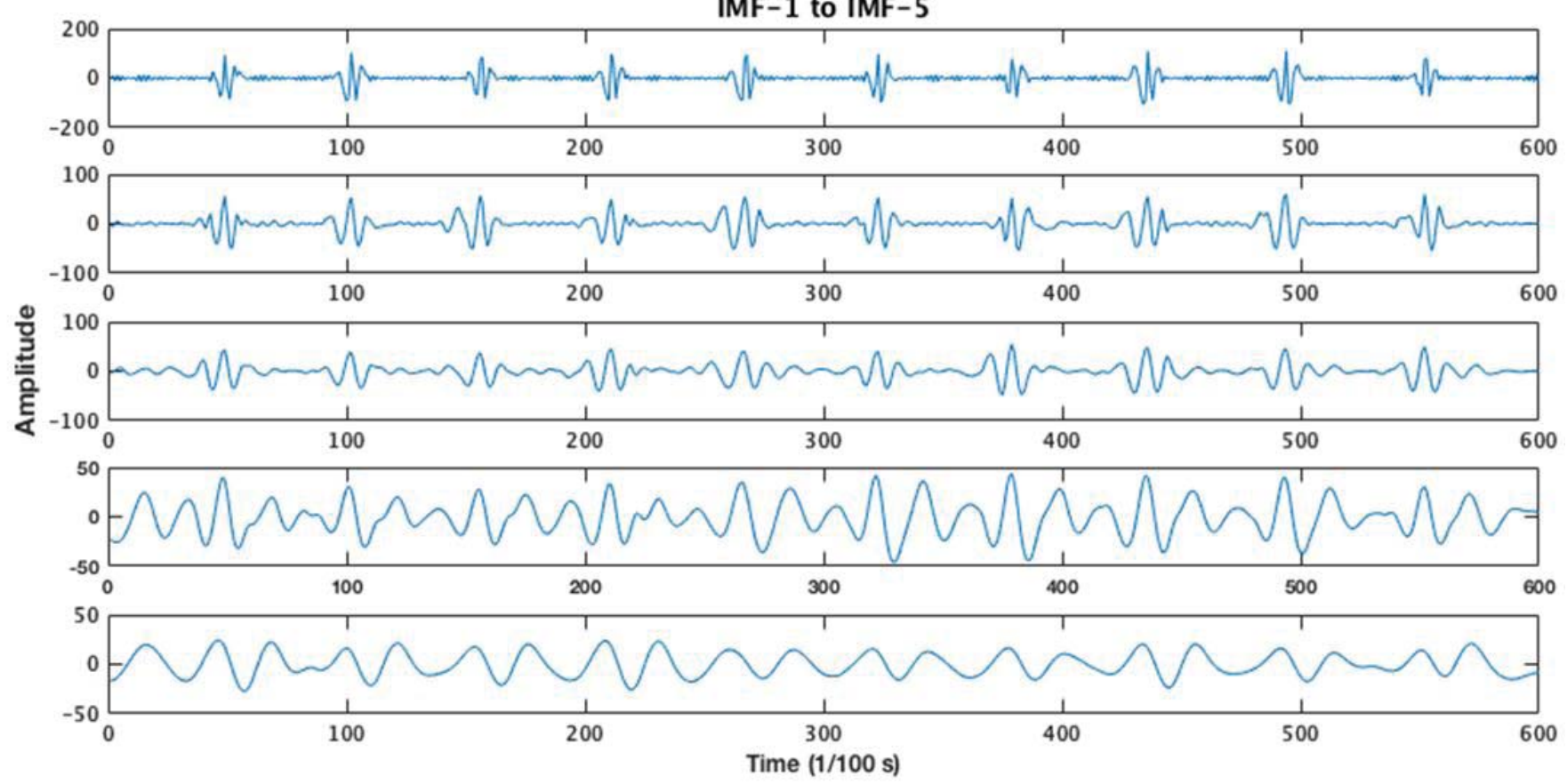

(a)

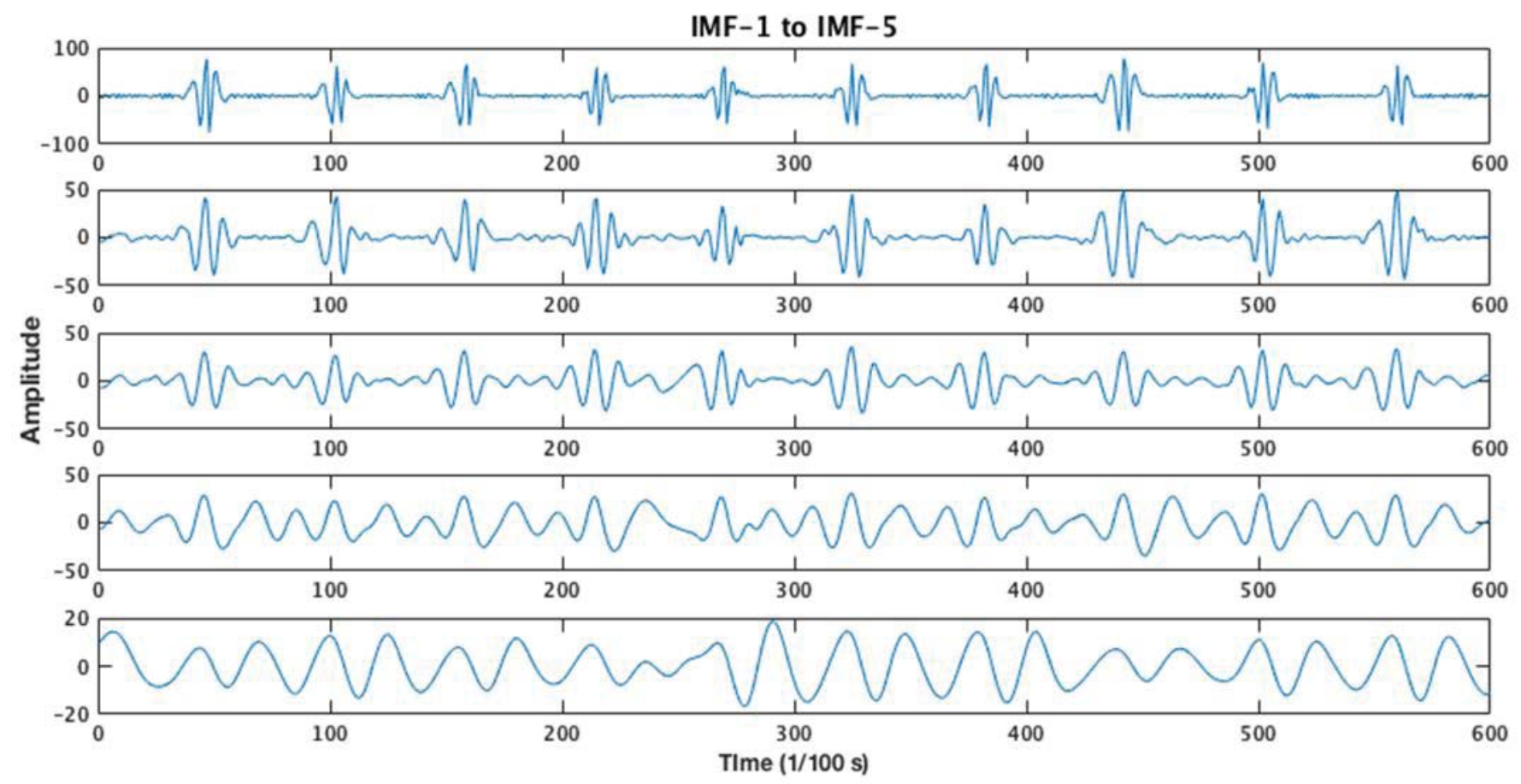

(b)

Figure 4: Decomposition signals using EEMD (a) subject-1 (b) subject-2

features with significant differences, especially in IMF-1, IMF-2, and IMF-4, significant differences in almost all features.In IMF-5, significant differences are only found in one feature. This can occur because a high-level IMF will generate relatively monotonous signals [41]. Since not all features have significant differences, in the validation process, all features are used as predictors, with expectations it can produce high accuracy. 

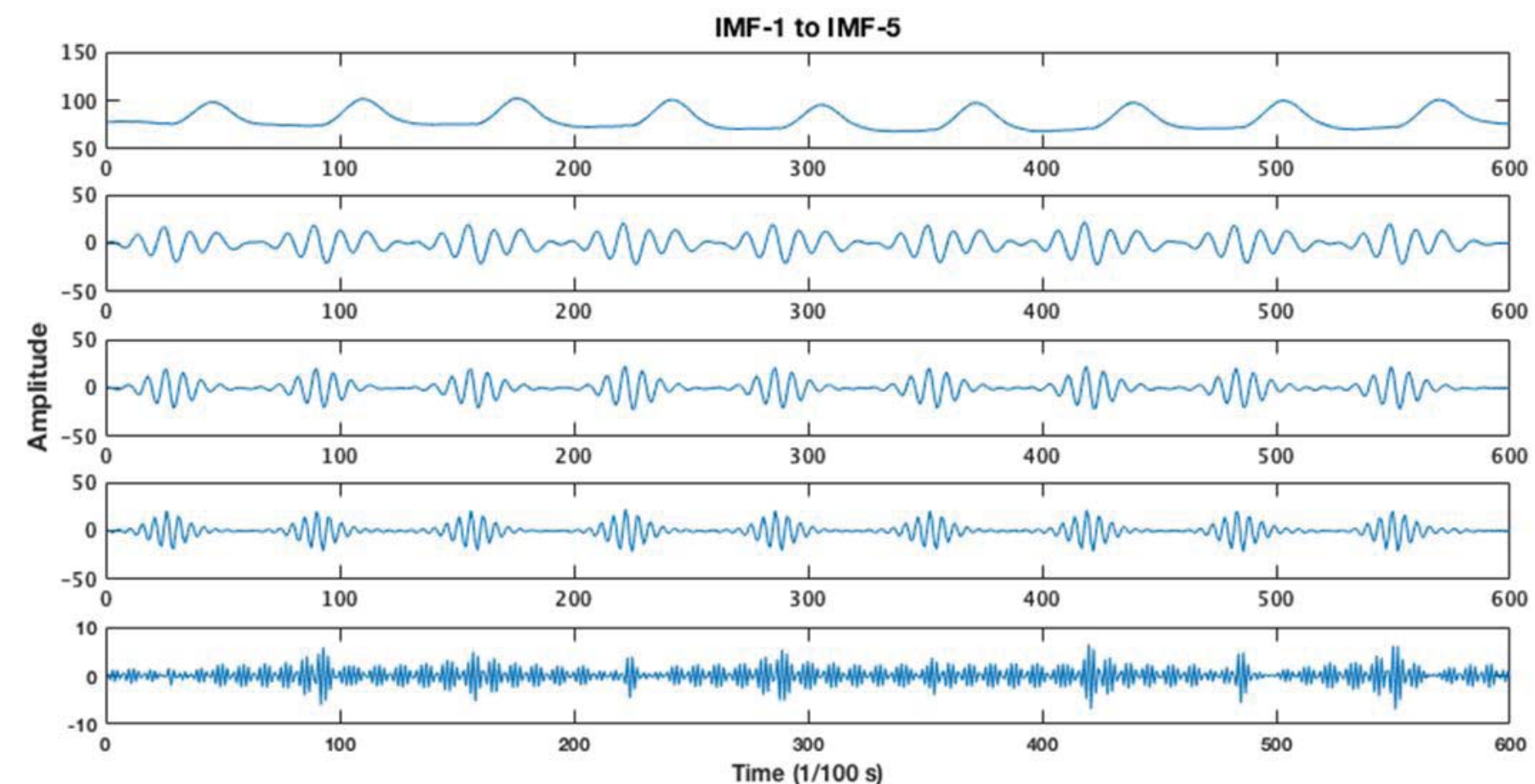

(a)

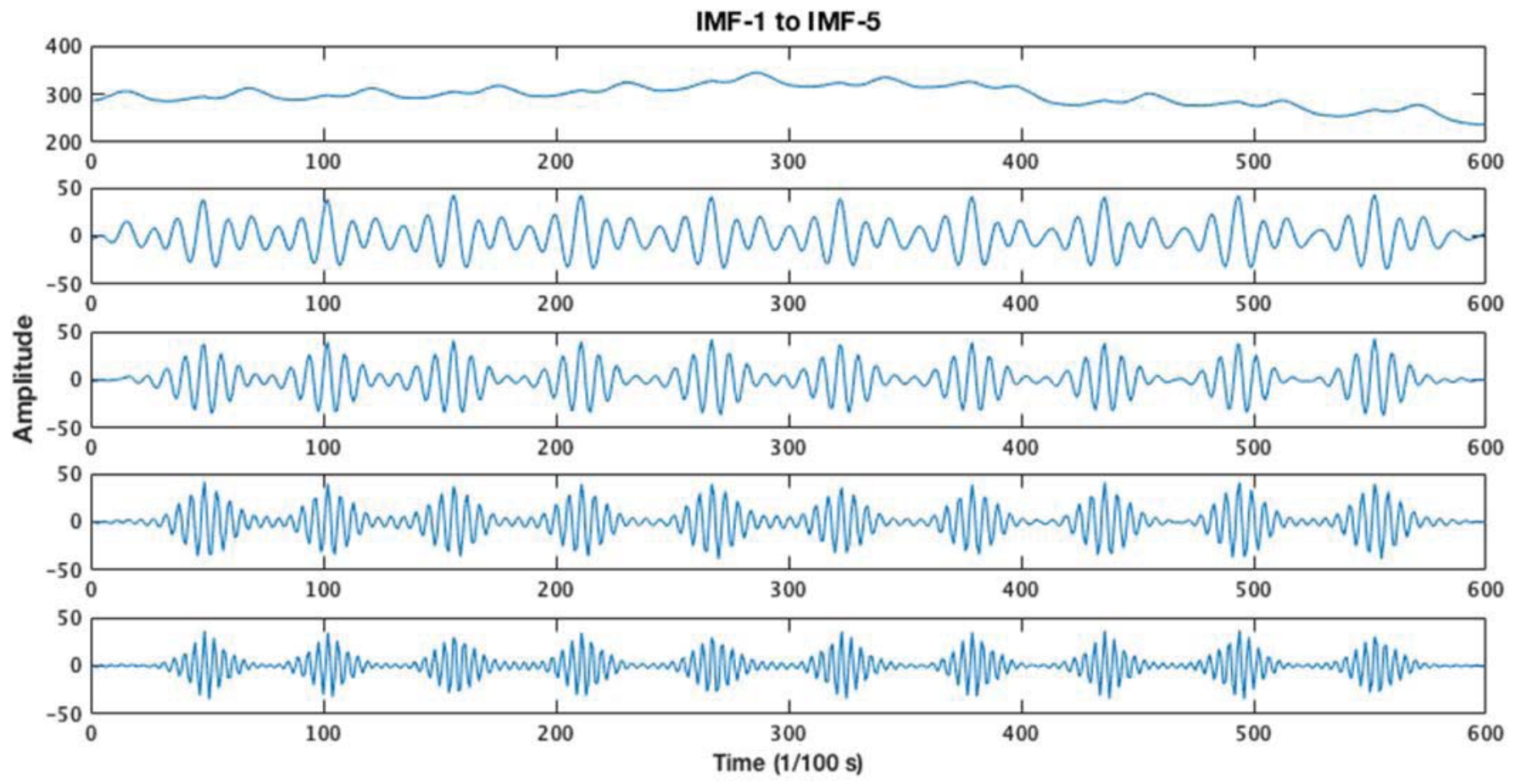

(b)

Figure 5: Decomposition signals using VMD (a) subject-1 (b) subject-2

The average value of each feature shown in Figure 6 represents that the subject's ECG signals have different characteristics from each other. As the results of the significance tests, which are shown in Table 1, visually in
Figure 6 shows that parameters such as mean, variance, skewness, and entropy have adjacent values. This might be considered in the feature selection strategy in order to get efficiency in the use of the number of features. 


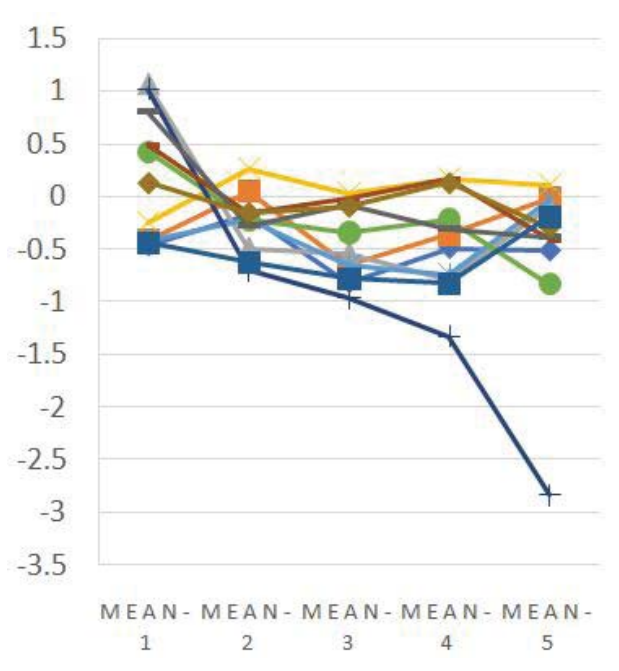

(a)

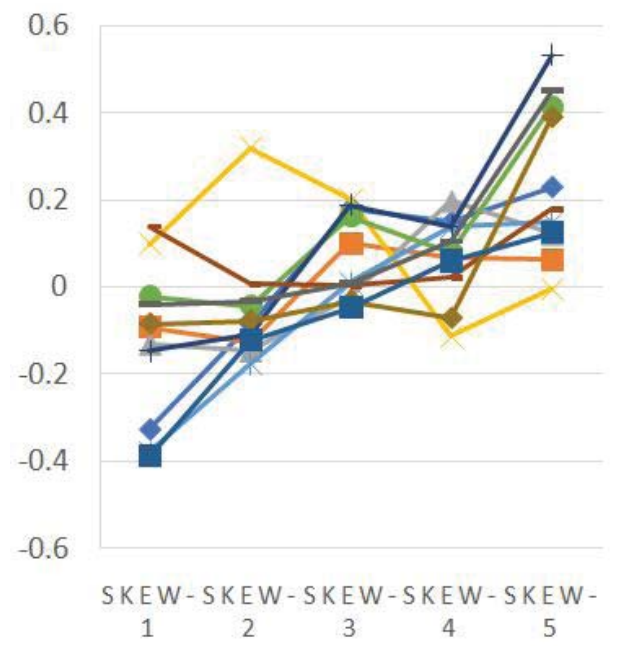

(c)

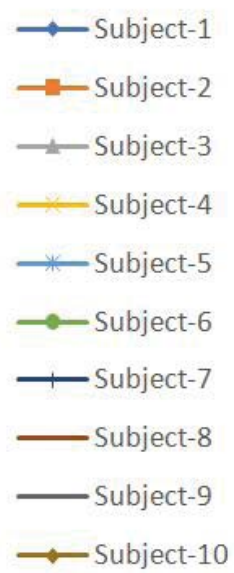

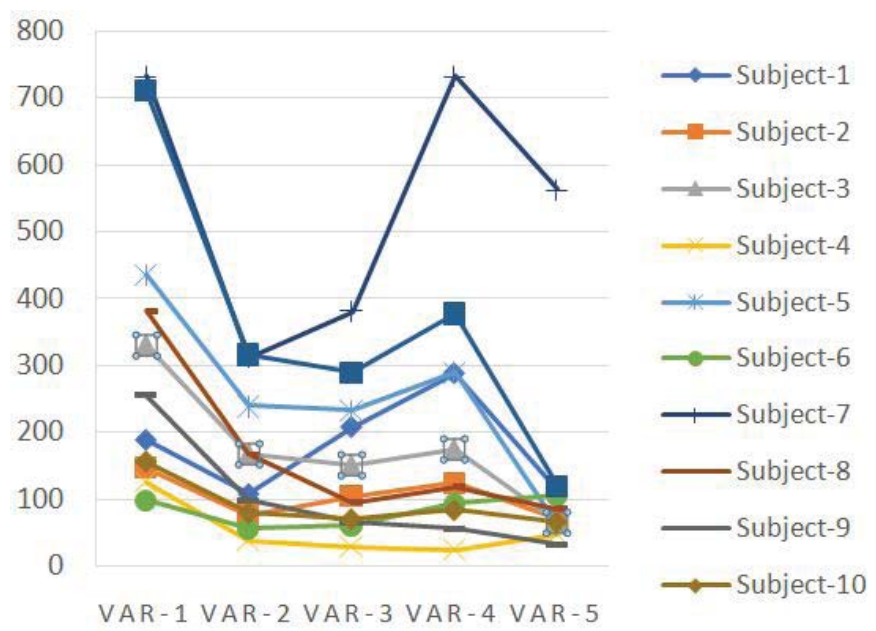

(b)

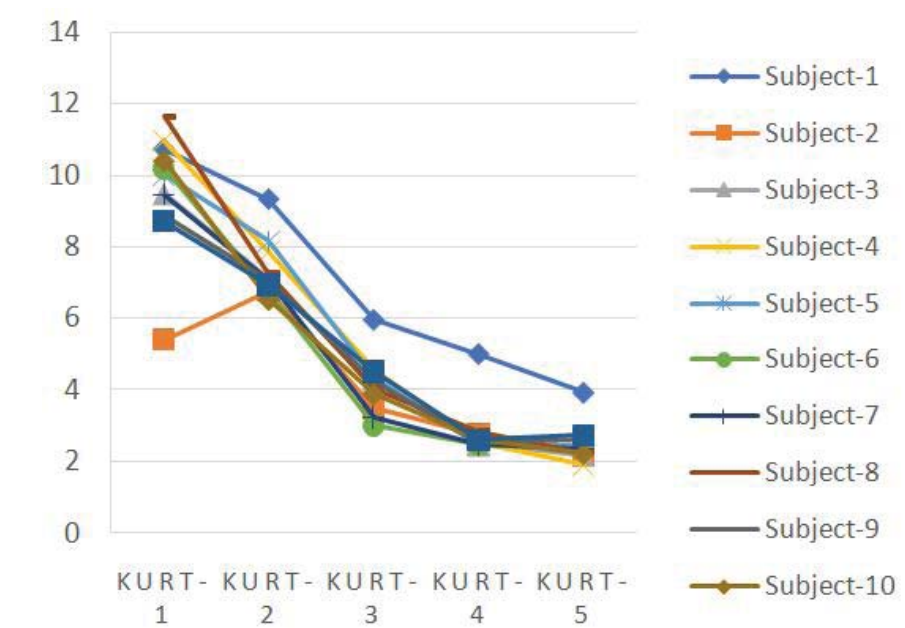

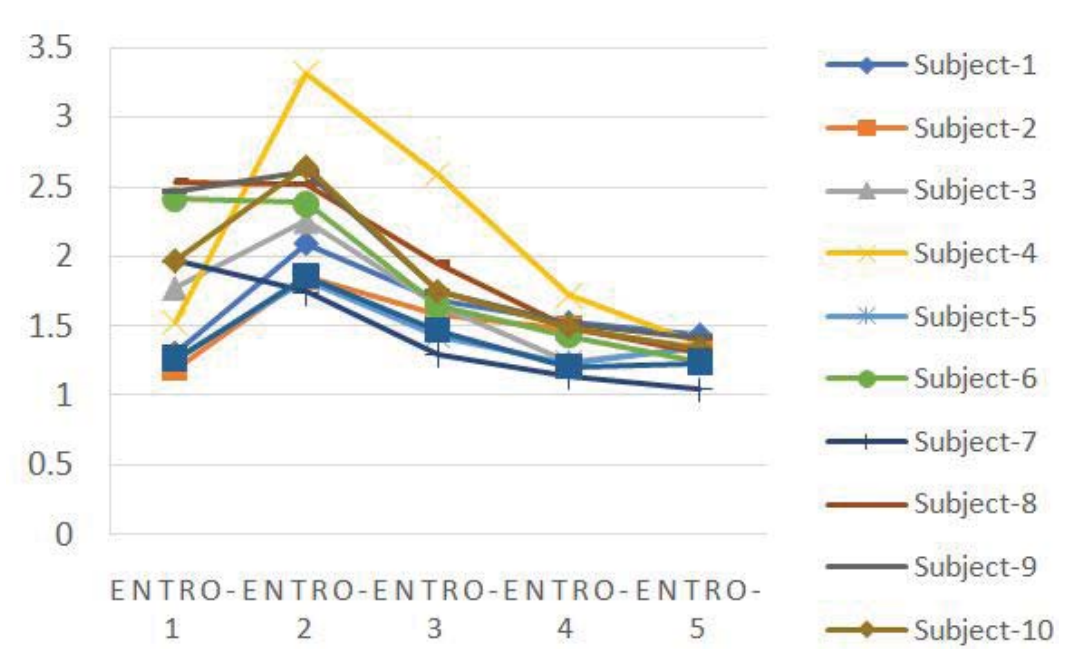

(d)

(e)

Figure 6: The average value of features (a) mean (b) variance (c) skewness (d) kurtosis (e) entropy of each IMF of decomposition (EEMD) 
The next stage is the validation of the proposed method. 10-fold cross-validation and SVM are used to validate the performance of the proposed method. Linear, quadratic, and cubic SVM are simulated to observe which kernels are capable of producing the highest accuracy and also generalize the performance of the proposed method. In this study, feature selection is not performed so that all attributes are used as predictors. The results of each simulation are shown in Table 2.

Table 2: Validation results of the proposed method

\begin{tabular}{|c|c|c|}
\hline \multirow{2}{*}{ Classifier } & \multicolumn{2}{|c|}{ Accuracy (\%) } \\
\cline { 2 - 3 } & EEMD & VMD \\
\hline Linear SVM & 98.2 & 98.2 \\
\hline Quadratic SVM & 98.2 & 98.2 \\
\hline Cubic SVM & 96.4 & 97.3 \\
\hline
\end{tabular}

Table 2 shows the highest accuracy was 98.2\%, both of EEMD and VMD. The highest accuracy is achieved by linear and quadratic SVM. In this case, the linear and quadratic kernels are able to produce higher accuracy than cubic kernels, about $0.9 \%$ to $1.8 \%$. Refers to the feature distribution pattern, which is shown in Figure 7 , linear or quadratic separation lines are the best boundary lines and also consider the efficiency of calculations compared to cubic kernel.

Table 3 confirms the results of the validation test in the confusion matrix format. It shows that the proposed methods are competitive due to the accuracy achieved and also give the same misidentification in subject- 1 and subject-3. This study outperforms some previous studies [18-20]. A summary of each study is shown in Table 4 . We specifically highlight the study [20], in which the method of signal decomposition and statistical feature extraction was also applied. We can conclude that EEMD and VMD have better performance compared to empirical mode decomposition as used in studies [20]. Theoretically, EEMD and VMD are able to decompose

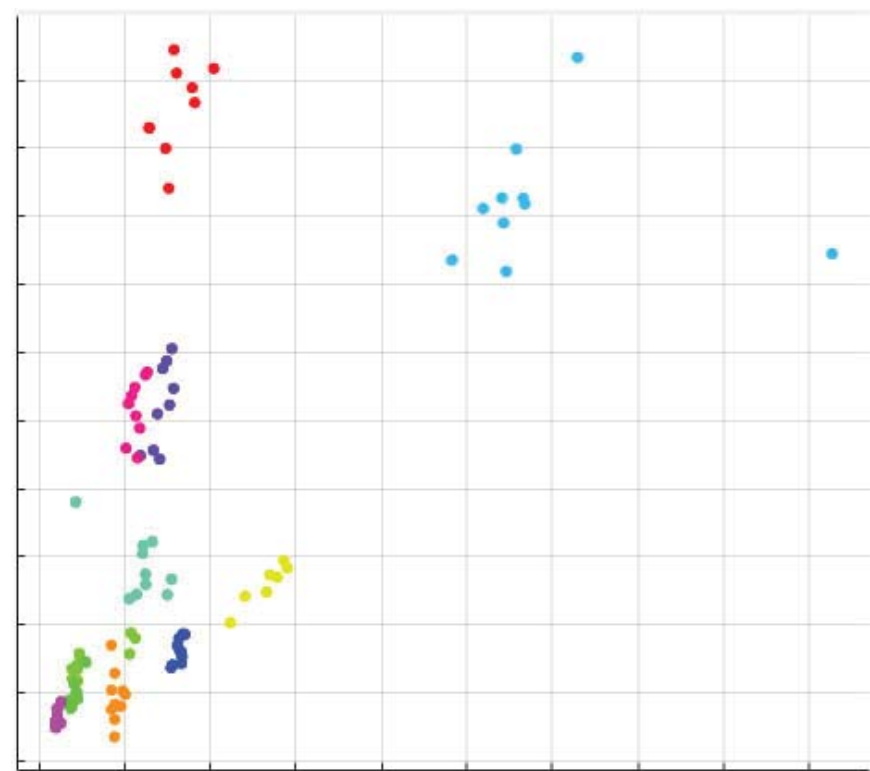

Figure 7: Pattern distribution of features for each subject

Table 3: Confusion Matrix for highest accuracy in both EEMD and VMD methods

\begin{tabular}{|c|c|c|c|c|c|c|c|c|c|c|c|c|c|}
\hline & \multicolumn{11}{|c|}{ Predicted Subject (Sn) } & \multirow{2}{*}{$\begin{array}{l}\text { Acc } \\
(\%)\end{array}$} \\
\hline & & $\mathrm{S}_{1}$ & $\mathrm{~S}_{2}$ & $\mathrm{~S}_{3}$ & $\mathrm{~S}_{4}$ & $\mathrm{~S}_{5}$ & $\mathrm{~S}_{6}$ & $\mathrm{~S}_{7}$ & $\mathrm{~S}_{8}$ & $\mathrm{~S}_{9}$ & $\mathrm{~S}_{10}$ & $\mathrm{~S}_{11}$ & \\
\hline \multirow{11}{*}{ 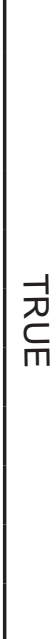 } & $\mathrm{S}_{1}$ & 9 & & & & & & 1 & & & & & 90 \\
\hline & $\mathrm{S}_{2}$ & & 10 & & & & & & & & & & 100 \\
\hline & $\mathrm{S}_{3}$ & & & 9 & 1 & & & & & & & & 90 \\
\hline & $\mathrm{S}_{4}$ & & & & 10 & & & & & & & & 100 \\
\hline & $\mathrm{S}_{5}$ & & & & & 10 & & & & & & & 100 \\
\hline & $\mathrm{S}_{6}$ & & & & & & 10 & & & & & & 100 \\
\hline & $\mathrm{S}_{7}$ & & & & & & & 10 & & & & & 100 \\
\hline & $\mathrm{S}_{8}$ & & & & & & & & 10 & & & & 100 \\
\hline & $\mathrm{S}_{9}$ & & & & & & & & & 10 & & & 100 \\
\hline & $\mathrm{S}_{10}$ & & & & & & & & & & 10 & & 100 \\
\hline & $\mathrm{S}_{11}$ & & & & & & & & & & & 10 & 100 \\
\hline
\end{tabular}

Table 4: Comparison of proposed methods with previous studies

\begin{tabular}{|c|c|c|c|c|}
\hline Reference & Number of subjects & Number of Dataset & Method & Accuracy (\%) \\
\hline$[18]$ & 10 & 65 & Samp En and Hjorth Descriptor & 93.8 \\
\hline$[19]$ & 11 & 110 & DWT and Entropy & 71.8 \\
\hline$[20]$ & 11 & 110 & EMD and Statistic & 93.6 \\
\hline Proposed method & 11 & $\mathbf{1 1 0}$ & EEMD, VMD and Statistic & $\mathbf{9 8 . 2}$ \\
\hline
\end{tabular}


the signal where each IMF of decomposition does not have a relationship with each other. This made the characteristics of the signal more specific. Therefore, this proposed method achieves better accuracy. Furthermore, it was confirmed in a study by Maji and Pal [42], that in certain cases, the VMD method produces better performance than EMD.

\section{CONCLUSIONS}

A personal identification using the new biometric approach based on single-lead ECG signals is proposed. This study is an improvement from previous research in order to get higher accuracy. In addition, this study used a larger population. A total of 110 ECG signals are taken from 11 participants were simulated in this study. The multilevel signal decomposition method, combined with the statistical calculation, is used for feature extraction. In the feature extraction stage, Ensemble Empirical Mode Decomposition (EEMD) and Variational Mode Decomposition (VMD) decompose the signal into five levels. Mean, variance, skewness, kurtosis, and entropy are calculated at each level of decomposition signal, which then becomes a set of features. Validation of proposed methods is done by 10 -fold cross-validation and SVM. Linear, quadratic, and cubic kernels were tested to find out the best performance. This research shows that the performance of EEMD and VMD is equally good, where both methods reach $98.2 \%$ accuracy. The results of this study generated an increase in accuracy of $4.4 \%$ compared to previous studies, which resulted in an accuracy of $93.8 \%$ in the same dataset. The increased accuracy also proves the hypothesis that ECG signals have unique characteristics among persons. In addition, the simulation provides evidence that EEMD and VMD have better performance than EMD. The same oscillation signal is not generated in different decomposition levels. It is very important to avoid bias in the feature extraction stage so that it impacts the detection accuracy. However, the biometric system that proposed in this study still has drawback including simulations carried out in small populations, offline simulation and have not tested the true acceptance rate and false acceptance rate. Thus, it remains a challenge for this new biometric approach can be applied in a real-world implementation.

\section{Data availability}

The data that support of this research are available upon request to corresponding author. Principal contact to sugondo@telkomuniversity.ac.id.

\section{Conflicts of interest}

We declare that we have no conflicts of interest in the authorship or publication of this contribution.

\section{REFERENCES}

1. Fratini, A., Sansone, M., Bifulco, P., \& Cesarelli, M. (2015). Individual identification via electrocardiogram analysis. BioMedical Engineering Online, 14(1), 1-23. https://doi.org/10.1186/s12938-015-0072-y

2. Nemirko A. P., Lugovaya T. S. (2005). Biometric human identification based on electrocardiogram. In Proc. XII-th Russian Conference on Mathematical Methods of Pattern Recognition (pp. 387-390). https://doi.org/10.1007/978-1-4614-7990-1

3. Biel, L., Pettersson, O., Philipson, L., \& Wide, P. (2001). ECG analysis: A new approach in human identification. IEEE Transactions on Instrumentation and Measurement, 50(3), 808-812. https://doi.org/10.1109/19.930458

4. Krasteva, V., Jekova, I., \& Schmid, R. (2018). Perspectives of human verification via binary QRS template matching of single-lead and 12lead electrocardiogram. PLoS ONE, 13(5), 1-25. https://doi.org/10.1371/journal.pone.0197240

5. Jekova, I., \& Bortolan, G. (2015). Personal Verification/Identification via Analysis of the Peripheral ECG Leads: Influence of the Personal Health Status on the Accuracy. BioMed Research International, 2015, 1-13. https://doi.org/10.1155/2015/135676

6. Belgacem, N., Nait-Ali, A., Fournier, R., \& Bereksi-Reguig, F. (2012). ECG Based Human Authentication using Wavelets and Random Forests. International Journal on Cryptography and Information Security, 2(2), 1-11. https://doi.org/10.5121/ijcis.2012.2201

7. Wei-quan, W., Pan, L. U., Jia-lun, L. I. N., \& Jin, Z. (2016). ECG Identification Based on Wavelet Transform. In Joint International Information Technology, Mechanical and Electronic Engineering Conference (JIMEC 2016) (pp. 497-501).

8. Pal, A., \& Singh, Y. N. (2019). Biometric recognition using area under curve analysis of electrocardiogram. International Journal of Advanced Computer Science and Applications, 10(1), 533-545. https://doi.org/10.14569/IJACSA.2019.0100169

9. Carreiras, C., Lourenco, A., Silva, H., \& Fred, A. (2013). A unifying approach to ECG biometric recognition using the wavelet transform. Lecture Notes in Computer Science (Including Subseries Lecture Notes in Artificial Intelligence and Lecture Notes in Bioinformatics), 7950 LNCS, 53-62. https://doi.org/10.1007/978-3-642-39094-4_7

10. Dar, M. N., Akram, M. U., Shaukat, A., \& Khan, M. A. (2015). ECG based biometric identification for population with normal and cardiac anomalies using hybrid HRV and DWT features. 2015 5th International Conference on IT Convergence and Security, ICITCS 2015 - Proceedings. https://doi.org/10.1109/ ICITCS.2015.7292977 
11. Chin, C. Y., Ramli, D. A., Chin, C. Y., Engineering, S. of E. and E., Campus, U. E., Malaysia, U. S., Malaysia. (2018). Development of Heartbeat Based Biometric System Using Wavelet Transform. Journal of Engineering Science, 14, 15-33. Retrieved from http://web.usm.my/jes/14_2018/JES_14_2018_2. pdf

12. Belgacem, N., Fournier, R., Nait-Ali, A., \& Bereksi-Reguig, F. (2015). A novel biometric authentication approach using ECG and EMG signals. Journal of Medical Engineering and Technology, 39(4), 226-238. https://doi.org/10.3109/03091902.2015.1021429

13. Belgacem, N., Amine Nait, A., \& Fethi, R. (2012). Person Identification System Based on Electrocardiogram Signal Using Lab VIEW. International Journal on Computer Science and Engineering, 4(06), 974-981.

14. Wang, D., Si, Y., Yang, W., Zhang, G., \& Li, J. (2019). A novel electrocardiogram biometric identification method based on temporal-frequency autoencoding. Electronics (Switzerland), 8(6), 1-24. https://doi. org/10.3390/electronics8060667

15. Odinaka, I., Lai, P. H., Kaplan, A. D., O'Sullivan, J. A., Sirevaag, E. J., Kristjansson, S. D., ... Rohrbaugh, J. W. (2010). ECG biometrics: A robust short-time frequency analysis. 2010 IEEE International Workshop on Information Forensics and Security, WIFS 2010. https://doi.org/10.1109/WIFS.2010.5711466

16. Arteaga-Falconi, J. S., Al Osman, H., \& El Saddik, A. (2016). ECG Authentication for Mobile Devices. IEEE Transactions on Instrumentation and Measurement, 65(3), 591-600. https://doi.org/10.1109/TIM.2015.2503863

17. Donida Labati, R., Munoz, E., Piuri, V., Sassi, R., \& Scotti, F. (2019). Deep-ECG: Convolutional Neural Networks for ECG biometric recognition. Pattern Recognition Letters, 126, 78-85. https://doi.org/10.1016/j.patrec.2018.03.028

18. Hadiyoso, S., Aulia, S., \& Rizal, A. (2019). OneLead Electrocardiogram for Biometric Authentication using Time Series Analysis and Support Vector Machine. International Journal of Advanced Computer Science and Applications, 10(2), 276-283. https://doi.org/10.14569/IJACSA.2019.0100237

19. Hadiyoso, S., Rizal, A., \& Wijayanto, I. (2019). ECG based biometric using wavelet packet decomposition. International Journal of Engineering and Advanced Technology, 9(1), 2178-2183.

20. Hadiyoso, S., Rizal, A., \& Aulia, S. (2019). ECG Based Person Authentication using Empirical Mode Decomposition and Discriminant Analysis. Journal of Physics: Conference Series, 1367, 1-10. https://doi.org/10.35940/ijeat.A9699.109119
21. Sigit, R., Hadiyoso, S., Rizal, A., \& Usman, K. (2014). Mini Wireless ECG for Monitoring Athletes' ECG Signal Based on Smartphone. IOSR Journal of Engineering, 4(6), 13-18. https://doi.org/10.9790/3021-04611318

22. Kwon, O., Jeong, J., \& Kim, H. Bin. (2018). ECG Sampling Frequency for HRV Analysis. Healthcare Informatics Research, 24(3), 198-206. https://doi.org/10.4258/hir.2018.24.3.198

23. Parak, J., \& Havlik, J. (2011). ECG signal processing and heart rate frequency detection methods. Proceedings of Technical Computing Prague, (January). Retrieved from http://amber2.feld.cvut.cz/bmeg/ wp-content/uploads/2012/03/Parak-TCP-2011.pdf

24. Lin, H., \& Chen, H. [2018]. Automated visual fault inspection of optical elements using machine vision technologies. Journal of Applied Engineering Science, 16(4), 447-453.

25. Yol, S., Ozdemir, M. A., Akan, A., \& Chaparro, L. F. (2018). Detection of Epileptic Seizures by the Analysis of EEG Signals Using Empirical Mode Decomposition. 2018 Medical Technologies National Congress, TIPTEKNO 2018, 1-4. https://doi.org/10.1109/TIPTEKNO.2018.8596780

26. Salmanvandi, M., \& Einalou, Z. (2017). Separation of twin fetal ECG from maternal ECG using empirical mode decomposition techniques. Biomedical Engineering - Applications, Basis and Communications, 29(6), 1-12. https://doi.org/10.4015/ S1016237217500429

27. Liu, G., \& Luan, Y. (2015). An adaptive integrated algorithm for noninvasive fetal ECG separation and noise reduction based on ICA-EEMD-WS. Medical and Biological Engineering and Computing, 53(11), 1113-1127. https://doi.org/10.1007/s11517015-1389-1

28. Wu, Z., \& Huang, N. E. (2009). Ensemble Empirical Mode Decomposition: a Noise-Assisted Data Analysis Method. Advances in Adaptive Data Analysis, 01(01), 1-41. https://doi.org/10.1142/ s1793536909000047

29. Dragomiretskiy, K., \& Zosso, D. (2014). Variational mode decomposition. IEEE Transactions on Signal Processing, 62(3), 531-544. https://doi.org/10.1109/ TSP.2013.2288675

30. Ye, H., Zhu, J., Cheng, Y., Xue, D., Wang, B., \& Peng, Y. (2018). PPG based Respiration Signal Estimation using VMD-PCA. In 2018 24th International Conference on Automation and Computing (ICAC)(pp. 1-5). https://doi.org/10.23919/iconac.2018.8748954

31. C. Cortes, \& Vapnik, V. (1995). Support Vector Networks. Machine Learning, 20(3), 273 - 297. https://doi.org/10.1007/BF00994018 
32. Victorovich, B. V., Sergeevich, T. V., Arkadievich, P. E., \& Anatolyevich, B. F. [2019]. Development of models for recognition of technological situations in the operation of electric centrifugal pumps for oil production. Journal of Applied Engineering Science, 17(4), 541-549

33. Jain, S., \& Salau, A. O. (2019). An image feature selection approach for dimensionality reduction based on kNN and SVM for AkT proteins. Cogent Engineering, 6(1), 1-14. https://doi.org/10.1080/23311916.20 19.1599537

34. Jain, S., \& Salau, A. O. (2019b). Detection of glaucoma using two dimensional tensor empirical wavelet transform. SN Applied Sciences, 1(11). https://doi.org/10.1007/s42452-019-1467-3

35. Smisek, R., Hejc, J., Ronzhina, M., Nemcova, A., Marsanova, L., Chmelik, J., Vitek, M. (2017). SVM Based ECG Classification Using Rhythm and Morphology Features, Cluster Analysis and Multilevel Noise Estimation, 44, 1-4. https://doi.org/10.22489/ CinC.2017.172-200

36. Smisek, R. (2016). ECG Signal Classification Based on SVM. Biomedical Engineering, (1), 365-369.

37. Wijayanto, I., Rizal, A., \& Hadiyoso, S. (2018). Multilevel Wavelet Packet Entropy and Support Vector Machine for Epileptic EEG Classification. In 2018 4th International Conference on Science and Technology (ICST) (pp. 9-14). Yogyakarta.
38. Wijayanto, I., Hartanto, R., \& Nugroho, H. A. (2020). Comparison of empirical mode decomposition and coarse-grained procedure for detecting pre-ictal and ictal condition in electroencephalography signal. Informatics in Medicine Unlocked, 19, 100325.https:// doi.org/10.1016/j.imu.2020.100325

39. Mitha, M., Shiju, S. S., \& Viswanadhan, M. (2014). Automated epileptic seizure detection using relevant features in support vector machines. 2014 International Conference on Control, Instrumentation, Communication and Computational Technologies, ICCICCT 2014, 1000-1004. https://doi.org/10.1109/ ICCICCT.2014.6993105

40. Boser, E., Vapnik, N., Guyon, I. M., \& Laboratories, T. B. (1992). Training Algorithm Margin for Optimal Classifiers. Perception, 144-152

41. Rizal, A., Hidayat, R., \& Nugroho, H. A. (2017). Lung Sound Classification Using Empirical Mode Decomposition and the Hjorth Descriptor. American Journal of Applied Sciences, 14(1), 166-173. https://doi. org/10.3844/ajassp.2017.166.173

42. Maji, U., \& Pal, S. (2016). Empirical mode decomposition vs. variational mode decomposition on ECG signal processing: A comparative study. In 2016 International Conference on Advances in Computing, Communications and Informatics, ICACCI 2016 (pp.1129-1134). https://doi.org/10.1109/ICACCl.2016.7732196
Paper submitted: 07.04.2020.

Paper accepted: 07.05.2020.

This is an open access article distributed under the CC $B Y-N C-N D 4.0$ terms and conditions. 